\title{
Acute effects of delta-9-tetrahydrocannabinol on performance monitoring in healthy volunteers
}

\author{
Desirée Spronk ${ }^{1,2}{ }^{*}$, Glenn J. H. Dumont ${ }^{1,3}$, Robbert J. Verkes ${ }^{1,2}$ and Ellen R. A. de Bruijn ${ }^{2}$ \\ 1 Department of Psychiatry, Radboud University Nijmegen Medical Centre, Nijmegen, Netherlands \\ 2 Donders Institute for Brain, Cognition and Behaviour, Radboud University Nijmegen, Nijmegen, Netherlands \\ ${ }^{3}$ Moleman Psychopharmacology, Amerongen, Netherlands
}

\section{Edited by:}

Patrizia Campolongo, Università degli

Studi di Roma La Sapienza, Italy

\section{Reviewed by:}

Gustav Schelling, Ludwig-Maximilians

University, Germany

Robert Hester, University of

Melbourne, Australia

Daniela Hauer, LMU Munich,

Germany

Jan R. Wessel, Max Planck Institute

for Neurological Research, Germany

\section{*Correspondence:}

Desirée Spronk, Department of

Psychiatry, Radboud University

Nijmegen Medical Centre, P.O. Box

9101, 6500 HB Nijmegen,

Netherlands.

e-mail:d.spronk@psy.umcn.nl

\begin{abstract}
Rationale: The error-related negativity (ERN) is a negative event-related potential that occurs immediately after an erroneous response and is thought to reflect human performance monitoring. Delta-9-Tetrahydrocannabinol (THC) administration in healthy volunteers has been linked to impaired performance monitoring in behavioral studies, but to date no studies have examined the effects of cannabinoids on the ERN. Methods: EEG data from 10 healthy volunteers was recorded during execution of a speeded choice-reactiontime task (Flankers task) after administration of THC or placebo vapor in a double-blind randomized crossover design. Results: The findings of this study show that the ERN was significantly reduced after administration of THC. The behavioral outcomes on the Flankers task showed no indications of drug-induced impairments. Discussion: The diminished ERN reflects impairments in the process of performance monitoring. The task design was not optimized to find behavioral effects. The study shows that cannabinoids impair performance monitoring.
\end{abstract}

Keywords: performance monitoring, error-related negativity, ERN, cannabinoids, THC, human, cannabis

\section{INTRODUCTION}

Several studies have shown that acute cannabis administration is associated with impairments of several cognitive processes (Gonzalez, 2007). One important process is the identification and correction of differences between intended and executed actions, also known as performance monitoring. This performancemonitoring system enables us to detect failures in our actions and to adapt our behavior accordingly. Therefore, it is an essential process for safe and efficient functioning in everyday situations. The functionality of the performance-monitoring system may vary with conditions such as fatigue, psychiatric disease, and drug taking (Scheffers et al., 1999; de Bruijn et al., 2004; Lorist et al., 2005; Schrijvers et al., 2009; Schellekens et al., 2010). Many drugs of abuse are known to increase the risk of engaging in maladaptive behavior, suggesting that drugs of abuse may impair human performance monitoring. Cannabis is the most frequently used illegal drug in Europe, most often self-administered for its mood-altering or "relaxing" effects (Green et al., 2003; Vicente et al., 2008). The use of cannabis and other cannabinoids for medical purposes as an analgesic or antiemetic for example is on the rise (Machado Rocha et al., 2008; Elikkottil et al., 2009). Surprisingly, to date the effect of cannabinoids on human performance monitoring is not sufficiently understood.

Cannabis contains a number of chemicals that belong to the class of cannabinoids. Delta-9-tetrahydrocannabinol (THC) is the main and most potent psychoactive cannabinoid of cannabis and is probably of greatest importance in the recreational use of the drug (Ashton, 1999; Russo and Guy, 2005). In pharmacological challenge studies in humans THC in isolation as well as cannabis has been administered. Administration of THC activates the cannabinoid receptors (CB1 and $\mathrm{CB} 2$ ). $\mathrm{CB} 1$ receptors are widespread in the brain, which probably accounts for the great variety of associated effects (Glass et al., 1997). These effects can be classified into two categories: affective and cognitive. Studies addressing the affective effects have shown that THC administration may cause an increase in anxiety and sedation and a decrease in motivation (Fusar-Poli et al., 2009; Dumont et al., 2011). Studies addressing the cognitive effects of THC, have often demonstrated that THC is associated with impairments in, e.g., working memory and attention (Crean et al., 2011). Studies of both human and animal subjects have also demonstrated that cannabis administration impairs behavioral flexibility and inhibitory control (McDonald et al., 2003; Ramaekers et al., 2006; Pattij et al., 2008).

Performance monitoring is a process that allows humans to respond actively and safely to changing environmental demands. Neural correlates of this process can be assessed by means of electroencephalography (EEG). When humans make an error in speeded choice-reaction tasks, a sharp negative peak is seen in the EEG around $50-100 \mathrm{~ms}$ after the erroneous response. Because of these characteristics, this event-related potential (ERP) component was named the error-related negativity (ERN; Falkenstein et al., 1990; Gehring et al., 1993). The ERN is considered a valid and reliable index of performance monitoring (Segalowitz et al., 2010). ERP recordings present a major advantage over behavioral outcomes, because ERP measures enable us to objectively investigate mechanisms underlying changes in cognitive functioning, for example as a result of drug effects (Kenemans and Kähkönen, 2011). Three influential theories have been developed that have 
thoroughly modeled the functional significance of the component: the Mismatch hypothesis (e.g., Falkenstein et al., 1991; Bernstein et al., 1995), the reinforcement-learning theory (RL; Holroyd and Coles, 2002), and the response-conflict theory (Yeung et al., 2004). The Mismatch hypothesis presumes that the ERN reflects a process that compares a representation of a correct response with the actual response. The RL-theory has been developed as an extension of the Mismatch Theory. According to the RL-theory the ERN reflects a learning process mediated by dopaminergic signaling in the mesencephalic dopaminergic nuclei when an outcome is worse than expected. The response-conflict theory, on the other hand, states that the ERN is generated when response-conflict occurs, i.e., in situations where a choice between several incompatible responses has to be made. Various imaging studies have implicated the anterior cingulate cortex (ACC) as the most likely candidate structure for generating the ERN (Herrmann et al., 2004; Stemmer et al., 2004; Debener et al., 2005). In line with this assumption, Debener et al. (2005) showed that larger ERN amplitudes are associated with a larger BOLD response in the ACC and that this is accompanied by stronger behavioral adaptations following errors.

To the authors' knowledge no previous studies have specifically addressed the effects of acute THC intoxication on the ERN. However, a number of other cognitive processes that are tightly coupled with performance monitoring have been investigated. First, Lane et al. (2005) found that cannabis decreases sensitivity to choice outcome during decision making tasks (Lane et al., 2005). The sensitivity to choice outcome can be interpreted as the behavioral consequence of performance monitoring. Second, working memory impairments following THC administration are probably one of the most consistently reported cognitive effects of THC (Ranganathan and D'Souza, 2006). Previously it was shown that working memory improvement was positively correlated with the ERN (Horowitz-Kraus and Breznitz, 2009). This coupling between working memory and performance monitoring also suggests that performance monitoring will be impaired after THC. Third, in a study on the long-term effects of cannabis use on error awareness was shown that regular cannabis users demonstrated less error awareness. Impaired error awareness is indicative of impaired performance monitoring. In the same report the authors also showed that this impairment was associated with hypoactivity in the ACC (Hester et al., 2009). Several imaging studies have shown that THC administration is associated with a reduction in cerebral blood flow in frontal brain regions (Borgwardt et al., 2008; Martín-Santos et al., 2010). Brain areas that are also of importance in performance monitoring. Together, these studies strongly suggest that THC administration is associated with compromised performance monitoring.

Jocham and Ullsperger (2009) mentioned in a recent review that investigating the effects of cannabinoids on the ERN is of particular relevance (Jocham and Ullsperger, 2009). They arrived at this conclusion because of the widespread distribution of cannabinoid receptors in the brain together with the growing use of THC. However, they also note in their review that to date these studies are lacking. Nonetheless, previous research can provide some hypotheses about the effects of cannabinoids on the ERN. Pharmacological studies, for example, have suggested that ERN characteristics depend on changes in dopaminergic neurotransmission. Specifically, in healthy volunteers the amplitude is increased after administration of the indirect dopaminergic agonist amphetamine (de Bruijn et al., 2004), and decreased by the dopamine 2 receptor antagonist haloperidol (Zirnheld et al., 2004; de Bruijn et al., 2006). Importantly, THC has also been shown to interact with the dopamine system, i.e., THC administration is followed by an increase in dopamine release in the striatum (Bossong et al., 2009). On this premise, it can be expected that ERN amplitudes are larger after THC administration.

The ERN may also be dependent on levels of motivation and sedation. Administration of alcohol or benzodiazepines (both substances known to induce sedation) has shown a reduction in the ERN amplitude (Johannes et al., 2001; Ridderinkhof et al., 2002; de Bruijn et al., 2004). Non-pharmacological studies have repeatedly demonstrated a positive correlation between ERN amplitude and motivation and arousal (de Bruijn et al., 2006; Ganushchak and Schiller, 2008). From this research it may be expected that THC may have a sedative and de-motivational effect which may reduce the ERN amplitudes post THC administration.

In summary, there may be two competing effects. Based on pharmacological studies we expect to observe an increased ERN following THC administration. Conversely, based on results from cognitive studies, we predict that THC will impair performance monitoring and that the ERN will therefore be reduced. At this point, we do not know which is the dominant effect. In order to investigate the effect of THC administration on the ERN, we subjected participants to the Flankers task after acute THC administration on two separate testing days in a placebo-controlled manner.

\section{MATERIAL AND METHODS SUBJECTS}

Sixteen healthy volunteers (12 male, four female), regular users between the ages of 18 and 27 were recruited through advertisement on the internet and at local drug testing services. All subjects met inclusion criteria of on average at least two exposures of THC per week in the last year and at least eight ecstasy exposures in the last 2 years. Detailed demographic data can be found in other reports (see e.g., Dumont et al., 2011). Exclusion criteria included pregnancy, (history of) psychiatric illness (assessed using the Structured Clinical Interview for DSM-IV axis I disorders, non-patient version (First et al., 1994) Axis II disorders were excluded using the Temperament and Character Inventory (Svrakic et al., 1993), use of over-the-counter medication within 2 months prior to the commencement of the study, (history of) treatment for addiction problems as assessed by a structured interview, excessive smoking ( $>10$ cigarets/day), and orthostatic dysregulation. Physical and mental health was determined by assessment of medical history, a physical, and electrocardiographic examination as well as standard hematological and chemical blood examinations. A total number of 10 subjects (eight male, two female, average age of 20.6 years) were included in the current analyses. Subjects smoked on average 4.6 exposures of THC per week for an average period of 5.9 years. Of the subjects excluded, one did not refrain from drug use, after which further study participation was denied. Two subjects experienced an adverse event that was judged to be likely related to study drug administration. 
Furthermore, for three subjects no EEG data could be analyzed due to technical problems. These six subjects were not included in the final data-analysis. All subjects provided their written informed consent before participating in the study, and were paid for their participation.

The study was approved by the Medical Ethics Committee of the Radboud University Nijmegen Medical Centre. The study is registered at The Netherlands Trial Registry (No. NTR1317).

\section{STUDY DRUGS}

THC was purified according to good manufacturing practice (GMP)-compliant procedures (Farmalyse BV, Zaandam, The Netherlands) from the flowers of Cannabis sativa grown under Good Agricultural Practice (Bedrocan BV Medicinal Cannabis, Veendam, The Netherlands; Choi et al., 2004; Hazekamp et al., 2004) and was dissolved in $200 \mu \mathrm{l} 100$ vol\% alcohol. THC was stored in a dark room at $-20^{\circ} \mathrm{C}$ in $1-\mathrm{ml}$ amber glass vials containing a Teflon screw cap secured with Parafilm to minimize evaporation. The $200-\mu 1$ 100\% alcohol solution without THC was used as placebo. On each study day, three subsequent dosages of $\mathrm{THC}(4,6$, and $6 \mathrm{mg})$ or placebo were administered at 90min intervals. Placebo and THC were administered by means of using a Volcano ${ }^{\circledR}$ vaporizer (Storz-Bickel GmbH, Tüttlingen, Germany), a validated method of intrapulmonary THC administration (Hazekamp et al., 2006; Abrams et al., 2007). Five minutes before administration, THC was vaporized at a temperature of $225^{\circ} \mathrm{C}$ and the vapor was stored in a polythene bag equipped with a valved mouthpiece, preventing the loss of THC in between inhalations. The transparent bag was covered with a black plastic bag to prevent unblinding. Subjects were not allowed to speak, and were instructed to inhale deeply and hold their breath for $10 \mathrm{~s}$ after each inhalation. Subjects were instructed to empty the bag within 2$3 \mathrm{~min}$. The inhalation procedure was practiced at screening using the mouthpiece of the vaporizer only.

\section{DESIGN AND PROCEDURE}

Placebo and THC were administered according to a balanced protocol in a randomized, double-blind, and crossover design. Every subject participated in both conditions with at least 7 days in between in which no other drug exposure was allowed. The current study was part of a larger study. Pharmacokinetic, cognitive, and neurophysiological data obtained from the study sample have been published previously (Dumont et al., 2009, 2011; Lansbergen et al., 2011).

To elicit ERNs, the participants performed a modified Flankers task (Eriksen and Eriksen, 1974; de Bruijn et al., 2004, 2006) in which they had to respond with either their left or right index finger to the central letter ( $\mathrm{H}$ or $\mathrm{S}$ ) of a congruent $\mathrm{HHHHH}$ or SSSSS) or incongruent (HHSHH or SSHSS) letter string. First, a fixation point was presented (lasting $100 \mathrm{~ms}$ ) followed $300 \mathrm{~ms}$ later by the stimulus (also lasting $100 \mathrm{~ms}$ ). During the next $900 \mathrm{~ms}$ the screen remained blank, after which a visual feedback stimulus appeared for $1000 \mathrm{~ms}$. The next trial was presented after an intertrial interval of $100 \mathrm{~ms}$. Visual feedback consisted of a yellow, a blue, or a red rectangle indicating whether the preceding response had been correct, incorrect or too late, respectively. Participants were instructed to respond as fast as possible to avoid feedback indicating that their response was too slow according to a preset reaction-time (RT) deadline. After written and verbal instructions, the participants familiarized themselves with the task in a practice block consisting of 60 trials, during which the initial RT deadline was set at a relatively liberal limit of $800 \mathrm{~ms}$. At the end of this practice block, the average RT and SD of the correct responses were computed. Next, for each individual participant and test day the RT deadline was determined by adding $0.5 \mathrm{SD}$ to the mean RT. For each subject and per each condition an individualized deadline was computed. Because previous studies on action monitoring have shown that ERN amplitude is affected by accuracy (see e.g., Gehring et al., 1993) including this RT deadline was essential to ensure that error rates did not vary across treatment conditions (de Bruijn et al., 2004, 2006). The experimental phase consisted of five blocks of 100 trials with a self-paced pause halfway through each block. After each block, participants were informed on the number of incorrect responses and responses whose latency exceeded the deadline. Verbal encouragement was given to keep performance accuracy around $80-90 \%$.

\section{PHARMACOKINETIC MEASUREMENTS}

Blood samples ( $4.5 \mathrm{ml}$ covered with aluminum foil) were taken at baseline 5, 20, 95, 110, 185, $200 \mathrm{~min}$ after the first THC administration. Plasma samples were immediately put on ice and were processed within $30 \mathrm{~min}$ after collection. Plasma samples were stored at a temperature of $-80^{\circ} \mathrm{C}$ for less than 3 months before laboratory analysis.

\section{EEG RECORDING}

The electroencephalogram (EEG) was recorded from 27 tin electrodes mounted in an elastic electrode cap (Electrocap International). Electrodes were placed at seven midline and 20 lateral locations in accordance with the international 10-20 system. All electrodes were referenced to the left mastoid. The vertical electrooculogram (EOG) was recorded bipolarly from electrodes placed above and below the right eye. The horizontal EOG was also recorded bipolarly from electrodes lateral to each eye. All electrode impedances were kept below $5 \mathrm{k} \Omega$ at the start of the recording session. The EEG and EOG signals were amplified using a timeconstant of $8 \mathrm{~s}$ and a bandpass between 0.02 and $30 \mathrm{~Hz}$. All signals were digitized with a sampling rate of $200 \mathrm{~Hz}$ using a 16-bit A/D converter.

\section{STATISTICAL ANALYSES}

Electro-oculogram artifact correction was carried out using the procedure proposed by Gratton et al. (1983). For the ERP analyses all responses with reaction times faster than $150 \mathrm{~ms}$ (placebo $1.5 \%$ and THC 1.0\%) were removed from the data sets. Epochs associated with correct and incorrect responses were averaged separately and time-locked to response onset, starting $100 \mathrm{~ms}$ before and ending $500 \mathrm{~ms}$ after response onset relative to a $100-\mathrm{ms}$ preresponse baseline. Correct responses were also averaged separately for congruent and incongruent stimuli time-locked to stimulus onset. The ERN was determined on correct and error trials in separate subject averages by subtracting the most negative peak in the 0 - to 200-ms time-window after response onset from the most positive peak in the time-window starting $80 \mathrm{~ms}$ before and 
ending $80 \mathrm{~ms}$ after response onset at electrode $\mathrm{FCz} / \mathrm{Cz}$, where ERN amplitude was largest (Holroyd et al., 2003; de Bruijn et al., 2004). The stimulus-locked ERPs were computed separately for correct congruent and incongruent trial types, in both treatment conditions. The amplitude of the N1 component was defined as the most negative deflection occurring in the 50 - to $150-\mathrm{ms}$ post stimulus time-window at electrodes FCz, Cz, and Pz. The N2 component was defined on incongruent trials as the most negative peak between 200-350 ms after stimulus onset at electrode FCz. The amplitude of the P300 was defined on incongruent stimuli as the largest positive deflection between 300 and $500 \mathrm{~ms}$ at electrodes $\mathrm{FCz}, \mathrm{Cz}$, and $\mathrm{Pz}$.

Individual averages for error rates and RTs were entered in a general linear model (GLM) with repeated measures (SPSS version 16.0, Chicago, IL, USA). The possible factors of the different GLMs were condition (two levels: THC or placebo), congruency (two levels: congruent vs. incongruent), and correctness (two levels: correct vs. incorrect). Adaptive behavior following erroneous responses was investigated by examining reaction times on correct responses following either correct or incorrect trials. To avoid serial congruency effects, only incongruent trials were included in these analyses. This type of performance adjustment is also known as post-error slowing (Rabbitt, 1966). A GLM analysis was performed with the factor condition (two levels: THC or placebo), and post-correctness (two levels: post-correct vs. post-error). The response-locked ERN was entered in a GLM, again with condition, congruency, and correctness as within subject factors. The stimulus-locked ERPs were analyzed by a GLM including condition (two levels: THC and placebo), congruency (congruent vs. incongruent), and electrode sites (three levels only for P300 and N1 analyses).

\section{RESULTS}

\section{THC PLASMA CONCENTRATIONS}

THC concentrations have previously been published (Dumont et al., 2011) but are reported here for the current sample selection. Average THC peak and trough plasma concentrations are shown in Table 1. THC concentrations during the placebo condition were always zero.

\section{BEHAVIORAL EFFECTS \\ Performance}

The percentage of Trial responses for each of the five possible response types for each condition and trial type is given in Table 2. The average error rate and average percentage of "too late" trial responses did not differ between the two drug conditions (both $p>0.1$ ). The ANOVA revealed that the error rate of incongruent trial types was higher than on congruent trial types $[F(1,9)=125.60, p<0.001]$. Similarly, there were more

Table 1 | Mean (SEM) THC peak (5 min after drug administration) and trough (20 min after drug administration) plasma levels (in $\mathrm{ng} / \mathrm{ml}$ ).

\begin{tabular}{|c|c|c|c|}
\hline & $4 \mathrm{mg}$ (1st) & $6 \mathrm{mg}(2 \mathrm{nd})$ & $6 \mathrm{mg}$ (3rd) \\
\hline Peak & $59.8(7.5)$ & 71.9 (10.9) & $89.2(18.0)$ \\
\hline Trough & $9.5(1.1)$ & $13.4(1.8)$ & $17.8(2.0)$ \\
\hline
\end{tabular}

"too late" responses at incongruent trials than at congruent trials $[F(1,9)=32.67, p<0.001]$. The interaction between congruency and condition did not reach significance for "incorrect" and "too late" trial responses $(p>0.05)$. The percentages for "too early" and "omission" responses showed that they constitute less than $4 \%$ of the responses in each condition.

\section{Reaction times}

A repeated measures ANOVA for correct and incorrect trials only ("too late" trials were excluded) showed that there were no differences between the placebo and THC condition on the reaction time (see Figure 1). There was a significant main effect of congruency $[F(1,9)=43.46, p<0.001]$ and correctness $[F(1,9)=66.39$, $p<0.001]$. Subjects were faster in general on the incorrect trials (314 $\mathrm{ms})$ in comparison to correct trials $(347 \mathrm{~ms})$ and performed faster on the congruent trials $(322 \mathrm{~ms})$ compared to incongruent trials $(339 \mathrm{~ms})$. No interaction effects were observed (all $p>0.1)$.

\section{Performance adjustments}

First, we compared reaction times of correct responses on trials that followed a correct response (post-correct) or an erroneous response (post-error). This post-error slowing analysis revealed neither a main effect for condition $[F(1,9)=0.11, p=0.743]$, nor

Table 2 | Mean percentages of correct, incorrect, too late, too early, and omission responses to congruent and incongruent trials for the placebo and THC condition.

\begin{tabular}{lccccc}
\hline & \multicolumn{2}{c}{ Congruent } & & \multicolumn{2}{c}{ Incongruent } \\
\cline { 2 - 3 } & Placebo & THC & & Placebo & THC \\
\hline \% Correct & 77.6 & 76.1 & & 52.8 & 53.7 \\
\% Incorrect & 12.5 & 15.3 & & 27.3 & 32.3 \\
\% Too late & 7.4 & 7.2 & & 16.6 & 12.1 \\
\% Too early & 1.4 & 1.0 & & 1.5 & 1.0 \\
\% Omission & 1.1 & 0.4 & & 1.8 & 0.9
\end{tabular}

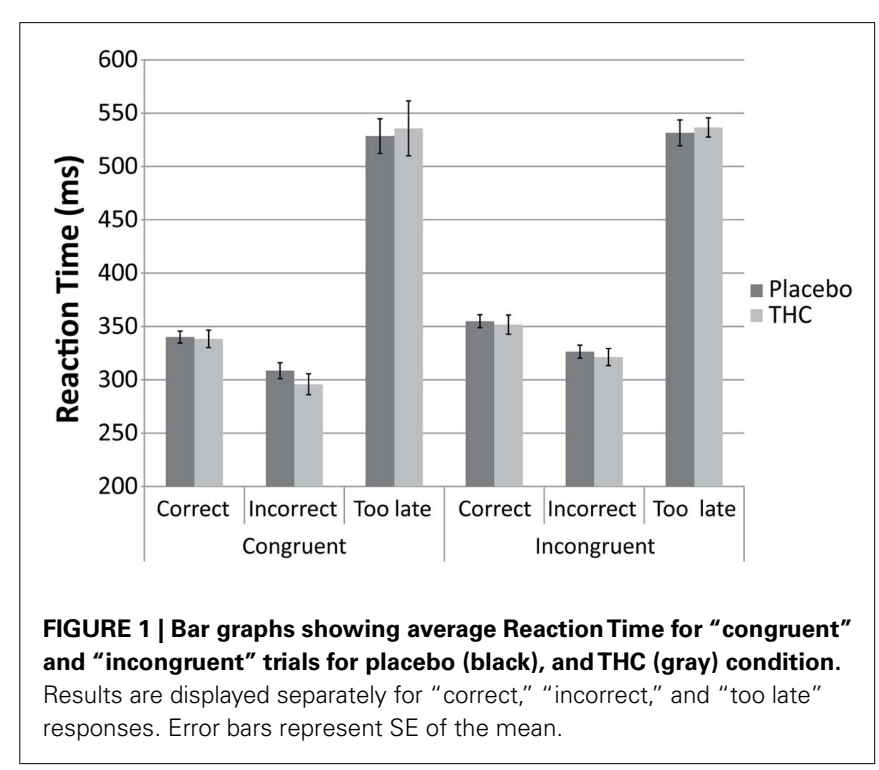


for post-correctness $[F(1,9)=2.48, p=0.150]$, nor an interaction between the two $[F(1,9)=0.92, p=0.362]$.

Second, we compared reaction times of correct responses that preceded an error (pre-error) or that followed an error (posterror). This post-error slowing analyses did reveal a main effect of post-error slowing $[F(1,9)=19.77, p=0.002]$. There was neither a significant main effect for condition $[F(1,9)=0.41, p=0.538]$, nor a significant interaction between the two $[F(1,9)=0.65$, $p=0.442]$. The main effect of post-error slowing showed that reaction times following an error $(349 \mathrm{~ms})$ were significantly slower than reaction times preceding the erroneous response (336 ms).

\section{ERP ANALYSES}

\section{Response-locked ERPs}

Figure 2 depicts the response-locked ERNs for the two treatment conditions. No overall significant effects of drugs were observed $[F(1,9)=0.072, p=0.795]$, nor was there a significant main effect of correctness $(p>0.1)$. There was a significant interaction between condition and correctness $[F(1,9)=7.00, p=0.027]$. Planned contrasts showed that the difference in the "ERN" for correct and incorrect trial responses was significant in the placebo condition $[F(1,9)=19.28, p=0.002,-0.9$ vs. $-4.9 \mu \mathrm{V}]$ but not in the THC condition $[F(1,9)=2.90, p=0.123,-2.4 \mu \mathrm{V}$ vs. $-3.9 \mu \mathrm{V}]$.

\section{STIMULUS-LOCKED ERPS}

To investigate whether the effects of THC on response-locked ERPs were not caused by an overall reduction in general stimulus processing or attention, additional stimulus-locked ERPs were conducted. Figure 3 depicts the grand average ERP wave for correct and incorrect trial responses separately for both conditions and for the three selected electrode sites. The waveform is in accordance with typical stimulus-locked waveforms.

\section{N1 amplitude}

For the N1 amplitude, the GLM only revealed a significant main effect of electrode $[F(1,9)=4.516, p=0.040]$. The post hoc tests showed that the effect was caused by larger N1 amplitudes at frontal and central sites $(-2.5$ and $-2.4 \mu \mathrm{V})$ in comparison to parietal sites $(-1.8 \mu \mathrm{V}, p<0.05)$. There was no effect of condition, nor a significant interaction effect between electrode and condition (all $p>0.1$ ).

\section{P300 amplitude}

For the P300 amplitude, there was only a significant main effect of electrode $[F(1,9)=6.829, p=0.023]$. The post hoc tests showed that the P300 amplitudes over the central and posterior electrode sites $(9.6$ and $10.1 \mu \mathrm{V})$ were significantly higher than over the frontal electrode site $(6,7 \mu \mathrm{V}, p<0.05)$. Drug condition had no effect on the P300 amplitude $(p>0.1)$.

\section{N2 amplitude}

The analyses on the N2 amplitude showed a main effect of congruency $[F(1,9)=18.575, p=0.002]$. As expected, the N2 amplitude was larger for incongruent trials than for congruent trials $(-2.7$ vs. $-0.9 \mu \mathrm{V})$. There was no main effect of condition, nor was there a condition by congruency interaction effect $(p>0.1)$.

\section{DISCUSSION}

The current study investigated the effects of THC administration on performance monitoring. Results showed that THC leads to reduced performance monitoring, as reflected in decreased ERN amplitudes compared to the placebo condition. The two conditions did not differ however, with respect to either behavioral performance measures or stimulus-locked ERP components.

\section{THC AND PERFORMANCE MONITORING}

Compromised performance monitoring as reflected by a reduced ERN under acute THC administration is consistent with a number of previous behavioral reports. For example, impairments in associated cognitive processes after THC and cannabis administration were demonstrated for reversal learning, inhibitory control, risk taking, and working memory (Curran et al., 2002; Ramaekers et al., 2006; Pattij et al., 2008; Hunault et al., 2009). We did not find any effects of condition on the behavioral measures of error rate, RT, and post-error slowing. The employment of individually determined RT deadlines results in a limited time-window in which participants are able to give a correct response. This procedure leads to a considerable limitation in the possible variance in performance and reaction times, but with the aim of maintaining similar performance levels between the conditions. The absence of an effect in performance measures is therefore not surprising and is a direct consequence of the individualized deadline. The reason we employed this method was to ensure that effects on the ERN would be due to the pharmacological condition, and not caused by differences in performance levels. This procedure is rather common in ERN studies as differences in performance may have an effect on ERN amplitude (see e.g., Gehring et al., 1993) and was employed in a number of other studies including from our own lab (e.g., Luu et al., 2000; de Bruijn et al., 2004, 2006; Debener et al., 2005). Comparable to our findings, in a number of other studies not always an association between the ERN and performance measures could be demonstrated (see e.g., Ullsperger et al., 2002; de Bruijn et al., 2004; Ullsperger and von Cramon, 2006). The absence of behavioral effects may, among other factors, be depending on sample size and the employed task design. The task design is likely to be the main contributing factor in our study. It is not unthinkable that employment of the Flankers task with different task parameters will yield behavioral effects in future experiments.

As stated in the introduction performance monitoring is a process that allows humans to respond actively and safely to changing environmental demands. Existing theories agree that this process reflected by the ERN is the result of a warning signal in the brain -error or conflict- that triggers the need for behavioral adaptation. In order to modify and improve behavior, other functions are recruited such as motor responses, attention, or learning. Although the relation with behavioral performance is often not that evident in highly controlled paradigms designed to investigate the ERN, the relevance of performance monitoring in daily life is evident. Everyday actions like safely driving a car require continuous performance monitoring and are obviously much more complex than choice-reaction tasks like the one currently used. Thus it is highly plausible that reductions in performance monitoring may be even more obvious in such complex behaviors. When drivers accidentally reach the verge of a road, they need to 

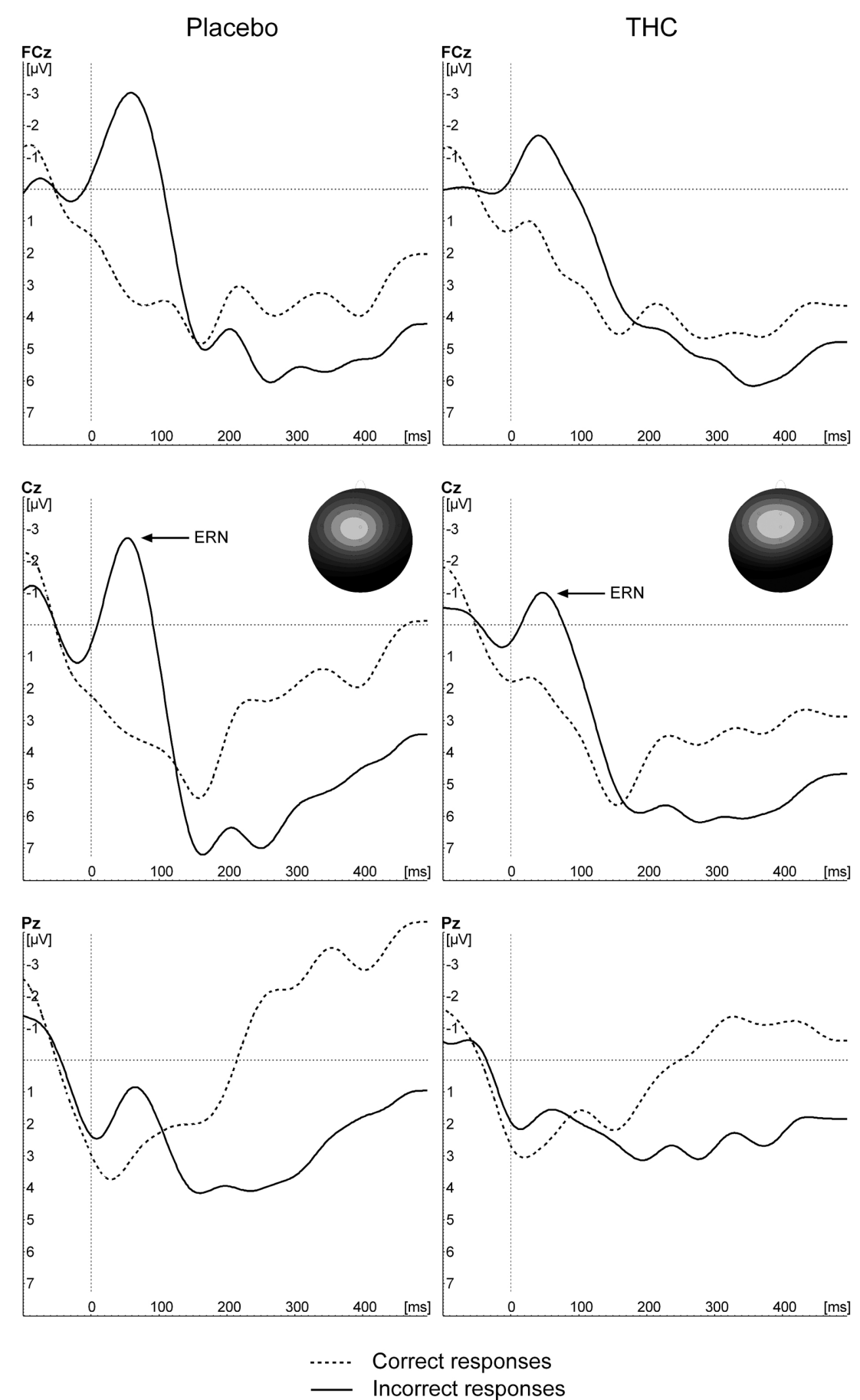

FIGURE 2 | Grand average waveforms of incorrect and correct responses to incongruent trial types for placebo and THC conditions.

recognize this and correct their steering in order to prevent the car from slipping off the road. Our results suggest that impairments in performance monitoring caused by THC administration may result in diminished warning signals and less efficient behavioral adaptations in a daily task like driving. In practice this could mean that the risk of slipping off the road is not timely notified and the required motor response to keep the car on the road is not operating correctly or fast enough. This suggestion is in line with recent findings from Calabria et al. (2010) and Penning et al. (2010) demonstrating that cannabis users show impaired driving abilities and have an increased risk to die in motor accidents.

In our study all subjects were regular users, i.e., at least 1-2 exposures per week in the last year. Also, the age range was small and all subjects had comparable durations of cannabis use. We 
Spronk et al.

THC and performance monitoring

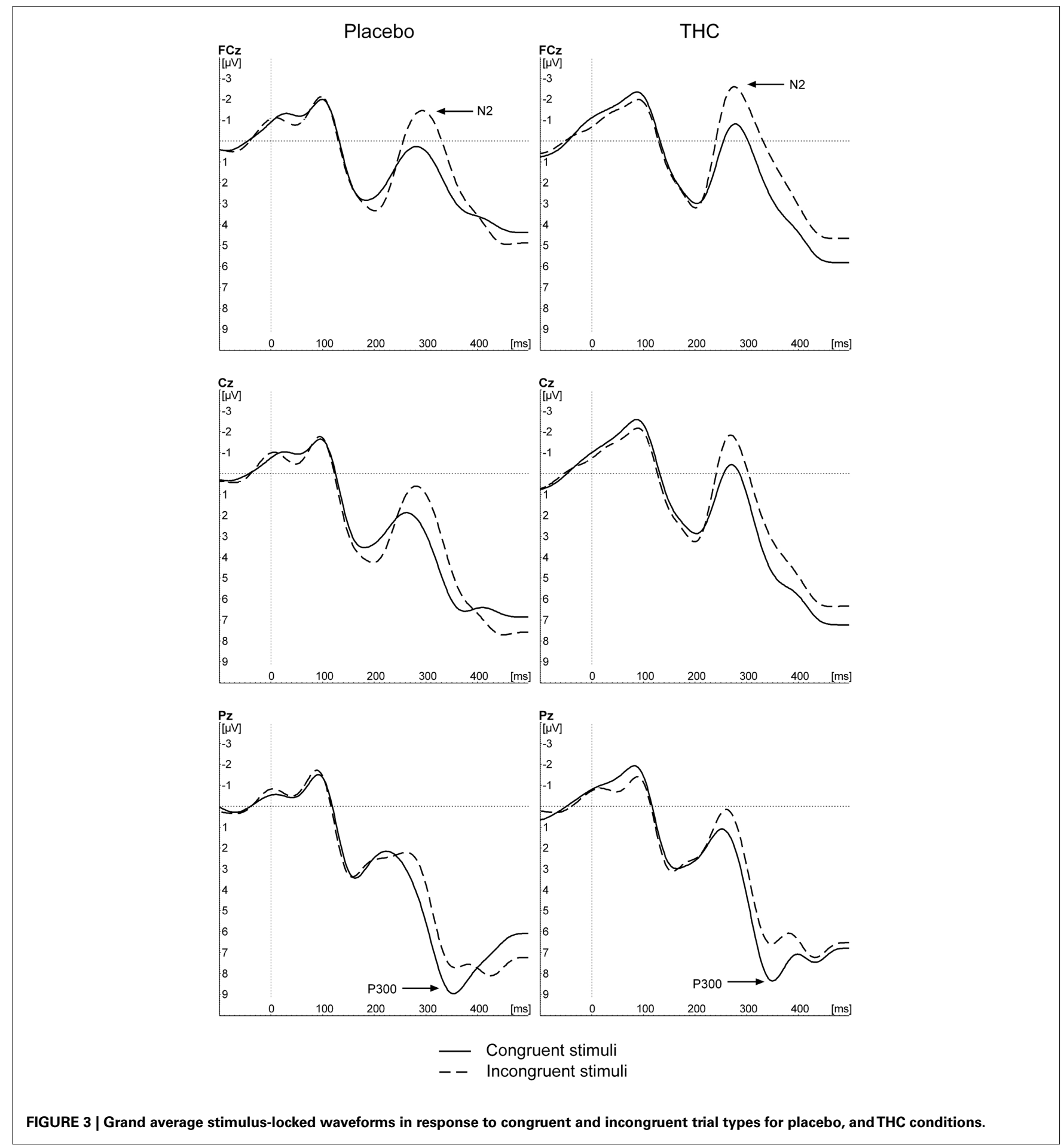

observed an effect of THC on performance monitoring in regular users, however, it is of interest if the effect is also observed in occasional cannabis users. Studies in which the effects of THC on performance monitoring are directly compared between orcasional and heavy users are warranted in order to directly investigate potential differences in affected cognitive processes. It is also imperative to compare acute drug effects with long-term drug effects in order to identify to what extent the cognitive profiles are different. For example, memory problems have repeatedly been found among heavy and long-term cannabis users, but may also occur under acute administration (see for a review Solowij and Battisti, 2008). It is also of importance to dissociate between acute drug effects in short-term occasional users vs. long-term/heavy users. For example, Ramaekers et al. (2009) compared the cognitine effects of THC administration between heavy and occasional users. They reported that THC significantly impaired performance

Frontiers in Behavioral Neuroscience

www.frontiersin.org

September 2011 | Volume 5 | Article 59 | 7 
on critical tracking, divided attention, and the stop signal task in occasional users, while in the heavy user group only stop signal performance was affected. Therefore, it is of importance to assess user history and to select subjects with comparable histories as this may interact with the cognitive process under investigation.

Another important question to address is to what extent the effect of THC on performance monitoring differs from other substances. It has been shown that alcohol and benzodiazepines also produce reductions in the ERN (de Bruijn et al., 2004; Ridderinkhof et al., 2002). In contrast to our results, benzodiazepine administration was associated with greater cognitive impairments as indicated by a slowed reaction time and absence of the N2 congruency effect. Despite control measures that were taken to ensure similar performance levels, benzodiazepine administration overruled this. In order to systematically address potential differences between THC and other pharmacological compounds, future studies in which THC, benzodiazepines, and alcohol are directly compared are recommended.

\section{PHARMACOLOGY}

Our study showed that activation of the cannabinoid system results in a reduction of the amplitude of the ERN. Previous studies have demonstrated ERN modulations by dopamine, i.e., DA agonists increase the amplitude and DA antagonists result in amplitude reductions (de Bruijn et al., 2004, 2006). THC administration is thought to increase dopaminergic release through disinhibition of GABAergic neurons (Pistis et al., 2002; Lupica et al., 2004), which implies an effect equivalent to a DA agonist. In keeping with previous pharmacological literature an increase in ERN amplitude would be expected, while we have observed the opposite in the present study. The dopamine system is also of importance in one of the three influential theories that have modeled the ERN: the RL-theory (Holroyd and Coles, 2002). The theory states that whenever a response is worse than expected, i.e., during commitment of an error, a negative error signal is generated which is coded as a phasic dopaminergic dip in the tonic activity of the mesencephalic dopaminergic system (Holroyd and Yeung, 2003). Holroyd and Yeung (2003) have outlined how the finding of the supposed increase in tonic mesencephalic dopaminergic neurotransmission by alcohol, may lead to a decreased ERN according to the RL-theory. One of the mechanisms they proposed is that increased tonic activity of the mesencephalic dopamine system, could lead to an increased inhibition of the ACC that in turn yields a reduction of the ERN. Similar to alcohol, cannabis also increases tonic dopaminergic neurotransmission in the mesencephalic brain areas (Boileau et al., 2003; Bossong et al., 2009). We therefore speculate that a similar mechanism occurs following THC administration.

The predictions from other pharmacological work and the RLtheory are contradictory and imply that there is a discrepancy within current opinions about dopaminergic pharmacology and the ERN/performance monitoring. Contributing to this conundrum is that drugs may affect dopaminergic neurotransmission via different pathways. Cannabis, e.g., may increase dopamine release via inhibition of the GABAergic system after activation of the endocannabinoid system. Amphetamine for example, interacts with dopamine by the redistribution of dopamine from the synaptic vesicle into the cytosol and the induction of reverse transport of dopamine through pre-synaptic reuptake transporters of dopamine through pre-synaptic reuptake transporters (Sulzer et al., 2005). We also do not sufficiently know how drugs induced changes in tonic mesencephalic dopamine neurotransmission relate to phasic dopaminergic in- and decreases and how this exactly translates to reinforcement-learning. Caution should thus be exercised in interpretation of our results in terms of the RL-theory. Future research into the underlying mechanisms of the RL-theory as well as the pharmacology of THC administration is needed.

Drugs rarely only affect dopaminergic neurotransmission, and this certainly also applies for administration of THC. Cannabinoid administration has also been associated with altered noradrenergic (Muntoni et al., 2006), GABAergic, and glutamatergic changes (Pistis et al., 2002). These other systems may also directly have an effect on the ERN. For example, it has been proposed that noradrenergic activation results in enlarged ERN amplitude (de Bruijn et al., 2004; Riba et al., 2005). This is also nicely illustrated with the example of alcohol administration, which is known to increase the release of GABA and of dopamine in the midbrain. Like THC, alcohol is associated with a reduction of the ERN amplitude (Ridderinkhof et al., 2002; Bartholow et al., in press). This example shows that it is hard to show which neurotransmission system the observed findings should be attributed to.

The endocannabinoid system has relatively recently been discovered and new perspectives and insights are booming. One new perspective is, for example, that THC administration in rats with a history of regular THC exposure yields a decrease in dopamine rather than an increase (Jentsch et al., 1998; Verrico et al., 2004). Although this preclinical work might not be directly comparable to the situation in our study, it is important to consider in the interpretation and discussion of our results in light of other pharmacological studies and the RL-theory. All subjects included in the current study used at least 1-2 cannabis exposures per week in the last year and can thus be considered as regular users. In order to better address this issue, it is highly recommended for future research to investigate if and how cannabis administration affects dopaminergic signaling in short vs. long-term users.

\section{MOTIVATION, ATTENTION, AND ALERTNESS}

To further explore the decreased ERN post THC administration, we evaluated three potential factors that could have influenced the decreased ERN. First, based on data obtained from a partial overlapping study sample, we previously published that THC administration causes a decrease in motivation (Dumont et al., 2011). These findings are in accordance with other reports (Böcker et al., 2010). Also, the ERN is known to be dependent on motivation levels (Bush et al., 2000; Boksem et al., 2006) and therefore a decrease in motivation levels could have indirectly modulated the observed reduction of the ERN. In order to address this with more objective measures we analyzed stimulus-locked ERPs. The amplitude of the stimulus-locked P300 component is most relevant for motivation, as its amplitude has previously been positively correlated with motivation (Nijboer et al., 2010). Despite the fact that no P300 differences could be found in our data, an effect of motivation cannot be excluded, because the self-report scales obtained from 
the same sample suggested that motivation decreased under THC affects. Similar to the effects of motivation, THC was shown to reduce attention and the ERN was previously shown to depend on subjects' attention levels (Pailing and Segalowitz, 2004; Böcker et al., 2010; Larson and Clayson, 2011). The N100 and P300 components are among the group of ERPs that are known to be reduced by decreased attention (Coull, 1998). Because we did not find an effect on these outcomes following THC administration, we could not provide support for the possibility that THC affects the ERN through reduction of attention.

Finally, we investigated the effect of sedation by analyzing the stimulus-locked N2 amplitude to congruent and incongruent trials. Previous work showed that this N2 congruency effect (i.e., increased conflict-induced N2 amplitudes on incongruent trials), is affected by strong sedative effects of drugs. Administration of benzodiazepines, for example, induces a reduction in this $\mathrm{N} 2 \mathrm{effect}$ (de Bruijn et al., 2004). We did not find an effect on the N2 after THC administration, which suggests our subjects were not heavily sedated. Alternatively, reduced N2 amplitude may be a specific biomarker of sedative effects of benzodiazepines and might not extrapolate to other sedative substances. Interestingly enough the administration of alcohol, which is also known to induce moderate sedative effects, also did not affect the amplitude of N2 (Ridderinkhof et al., 2002). Saccadic eye movement can also be used to measure sedation. Again, previously published data of a partly overlapping subject sample showed no effects of THC on saccadic eye-movements (Dumont et al., 2011). In contrast, the subjective alertness scale showed a significant decrease in the THC condition (Dumont et al., 2011). Taken together, the subjective measures suggest that the ERN could be mediated by sedation. However, this could not be supported by the objective measures and thus suggests

\section{REFERENCES}

Abrams, D. I., Vizoso, H. P., Shade, S. B., Jay, C., Kelly, M. E., and Benowitz, N. L. (2007). Vaporization as a smokeless cannabis delivery system: a pilot study. Clin. Pharmacol. Ther. 82, 572-578.

Ashton, C. H. (1999). Adverse effects of cannabis and cannabinoids. $\mathrm{Br}$. J. Anaesth. 83, 637-649.

Bartholow, B. D., Henry, E. A., Lust, S. A., Saults, J. S., and Wood, P. K. (in press). Alcohol effects on performance monitoring and adjustment: affect modulation and impairment of evaluative cognitive control. J. Abnorm. Psychol. doi: 10.1037/a0023664. [Epub ahead of print].

Bernstein, P. S., Scheffers, M. K., and Coles, M. G. H. (1995). "Where did I go wrong?” A psychophysiological analysis of error detection. J. Exp. Psychol. Hum. Percept. Perform. 21, 1312-1322.

Böcker, K. B., Gerritsen, J., Hunault, C. C., Kruidenier, M., Mensinga, T. T., and Kenemans, J. L. (2010). Cannabis with high $89-\mathrm{THC}$ contents affects perception and visual selective attention acutely: an event-related potential study. Pharmacol. Biochem. Behav. 96, 67-74.

Boileau, I., Assaad, J. M., Pihl, R. O., Benkelfat, C., Leyton, M., Diksic, M., Tremblay, R. E., and Dagher, A. (2003). Alcohol promotes dopamine release in the human nucleus accumbens. Synapse 49, 226-231. Lorist, M. M. (2006). Mental fatigue, motivation and action monitoring. Biol. Psychol. 72, 123-132.

Borgwardt, S. J., Allen, P., Bhattacharyya, S., Fusar-Poli, P., Crippa, J. A., Seal, M. L., Fraccaro, V., Atakan, Z., Martin-Santos, R., O'Carroll, C., Rubia, K., and McGuire, PK. (2008). Neural basis of delta-9-tetrahydrocannabinol and cannabidiol: effects during response inhibition. Biol. Psychiatry 64, 966-973.

Bossong, M. G., van Berckel, B. N., Boellaard, R., Zuurman, L., Schuit, R. C., Windhorst, A. D., van Gerven, J. M., Ramsey, N. F., Lammertsma,
Boksem, M. A., Meijman, T. F., and a discrepancy between the two. Consequently, more research should be conducted in this area to better address the sedative drug effects and their relation with performance monitoring.

\section{CONCLUSION}

To conclude, our findings suggest that administration of THC has a diminishing effect on human performance monitoring as reflected by reduced ERN amplitudes. Given the small size of the study consisting of only 10 subjects, the results should be considered as preliminary and need to be confirmed with larger samples. Nevertheless, the results are relevant for several reasons. First, THC is the most important component of cannabis, which is a drug that is recreationally used by many people over the world. The study provides a better understanding of the risks of cannabis use during performance of complex functions like driving which require a high level of performance monitoring. Second, as THC is increasingly examined and applied for clinical applications, mapping the potential (cognitive) side-effects are crucial aspects of patient's safety and drug compliance. We for the first time demonstrated that activation of the endocannabinoid system influences the ERN. We believe that the results of this study have extended our understanding of the cognitive effects associated with cannabinoids. The effects of cannabinoids on performance monitoring and cognitive process in general, need further evaluation.

\section{ACKNOWLEDGMENTS}

This work was supported by a grant received from ZonMW (31000062) to Robbert J. Verkes. Ellen R. A. de Bruijn is supported by a VENI grant (451-07-022) from the Netherlands Organization for Scientific Research (NWO). We thank Jan Leijtens and Roos Reitsma for their assistance in data collection.
A. A., and Kahn, R. S. (2009). Delta 9-tetrahydrocannabinol induces dopamine release in the human striatum. Neuropsychopharmacology 34, 759-766.

Bush, G., Luu, P., and Posner, M. I. (2000). Cognitive and emotional influences in anterior cingulate cortex. Trends Cogn. Sci. (Regul. Ed.) 4, 215-222.

Calabria, B., Degenhardt, L., Hall, W., and Lynskey, M. (2010). Does cannabis use increase the risk of death? Systematic review of epidemiological evidence on adverse effects of cannabis use. Drug Alcohol Rev. 29, 318-330.

Choi, Y. H., Hazekamp, A., PeltenburgLooman, A. M., Frédérich, M., Erkelens, C., Lefeber, A. W., and Verpoorte, R. (2004). NMR assignments of the major cannabinoids and cannabiflavonoids isolated from flowers of Cannabis sativa. Phytochem. Anal. 15, 345-354.

Coull, J. T. (1998). Neural correlates of attention and arousal: insights from electrophysiology, functional neuroimaging and psychopharmacology. Prog. Neurobiol. 55, 343-361.

Crean, R. D., Crane, N. A., and Mason, B. J. (2011). An evidence based review of acute and long-term effects of cannabis use on executive cognitive functions. J. Addict. Med. 5, $1-8$.

Curran, H. V., Brignell, C., Fletcher, S., Middleton, P., and Henry, J. (2002). Cognitive and subjective dose-response effects of acute oral Delta 9-tetrahydrocannabinol (THC) in infrequent cannabis users. Psychopharmacology (Berl.) 164, 61-70.

de Bruijn, E. R. A., Hulstijn, W., Verkes, R. J., Ruigt, G. S. F., and Sabbe, B. G. C. (2004). Drug-induced stimulation and suppression of action monitoring in healthy volunteers. Psychopharmacology (Berl.) 177, 151-160.

de Bruijn, E. R. A., Sabbe, B. G., Hulstijn, W., Ruigt, G. S. F., and Verkes, R. J. (2006). Effects of antipsychotic and antidepressant drugs on action monitoring in healthy volunteers. Brain Res. 1105, 122-129. 
Debener, S., Ullsperger, M., Siegel, M., Fiehler, K., von Cramon, D. Y., and Engel, A. K. (2005). Trial-bytrial coupling of concurrent electroencephalogram and functional magnetic resonance imaging identifies the dynamics of performance monitoring. J. Neurosci. 25, 11730-11737.

Dumont, G. J. H., Kramers, C., Sweep, F. C., Touw, D. J., van Hasselt, J. G., de Kam, M., van Gerven, J. M., Buitelaar, J. K., and Verkes, R. J. (2009). Cannabis coadministration potentiates the effects of "ecstasy" on heart rate and temperature in humans. Clin. Pharmacol. Ther. 86, 160-166.

Dumont, G. J. H., van Hasselt, J. G., de Kam, M., van Gerven, J. M., Touw, D. J., Buitelaar, J. K., and Verkes, R. J. (2011). Acute psychomotor, memory and subjective effects of MDMA and THC co-administration over time in healthy volunteers. J. Psychopharmacol. (Oxford) 25, 478-489.

Elikkottil, J., Elikottil, J., Gupta, P., and Gupta, K. (2009). The analgesic potential of cannabinoids. J. Opioid Manag. 5, 341-357.

Eriksen, B. A., and Eriksen, C. W. (1974). Effects of noise letters upon the identification of a target letter in a nonsearch task. Percept. Psychophys. 16, 143-149.

Falkenstein, M., Hohnsbein, J., Hoormann, J., and Blanke, L. (1990). "Effects of errors in choice reaction tasks on the ERP under focused and divided attention," in Psychophysiological Brain Research, eds C. H. M. Brunia, A. W. K. Gaillard, and A. Kok (Tilburg: Tilburg University Press), 192-195).

Falkenstein, M., Hohnsbein, J., Hoormann, J., and Blanke, L. (1991). Effects of crossmodal divided attention on late ERP components. II. Error processing in choice reaction tasks. Electroencephalogr. Clin. Neurophysiol. 78, 447-455.

First, M. B., Frances, A. J., Pincus, H., Vettorello, N., and Davis, W. W. (1994). DSM-IV in progress. Changes in substance-related, schizophrenic, and other primarily adult disorders. Hosp. Community Psychiatry 45, 18-20.

Fusar-Poli, P., Crippa, J. A., Bhattacharyya, S., Borgwardt, S. J., Allen, P., Martin-Santos, R., Seal, M., Surguladze, S. A., O'Carrol, C., Atakan, Z., Zuardi, A. W., and McGuire, P. K. (2009). Distinct effects of 89 -tetrahydrocannabinol and cannabidiol on neural activation during emotional processing. Arch. Gen. Psychiatry 66, 95-105.

Ganushchak, L. Y., and Schiller, N. O. (2008). Motivation and semantic context affect brain error-monitoring activity: an event-related brain potentials study. Neuroimage 39, 395-405.

Gehring, W. J., Goss, W., Coles, M. G. H., Meyer, D. E., and Donchin, E. (1993). A neural system for error detection and compensation. Psychol. Sci. 4, 385-390.

Glass, M., Dragunow, M., and Faull, R. L. (1997). Cannabinoid receptors in the human brain: a detailed anatomical and quantitative autoradiographic study in the fetal, neonatal and adult human brain. Neuroscience $77,299-318$.

Gonzalez, R. (2007). Acute and nonacute effects of cannabis on brain functioning and neuropsychological performance. Neuropsychol. Rev. 17, 347-361.

Gratton, G., Coles, M. G., and Donchin, E. (1983). A new method for offline removal of ocular artifact. Electroencephalogr. Clin. Neurophysiol. 55, 468-484.

Green, B., Kavanagh, D., and Young, R. (2003). Being stoned: a review of self-reported cannabis effects. Drug Alcohol Rev. 22, 453-460.

Hazekamp, A., Choi, Y. H., and Verpoorte, R. (2004). Quantitative analysis of cannabinoids from Cannabis sativa using 1H-NMR. Chem. Pharm. Bull. 52, 718-721.

Hazekamp, A., Ruhaak, R., Zuurman, L., van Gerven, J., and Verpoorte, R. (2006). Evaluation of a vaporizing device (Volcano) for the pulmonary administration of tetrahydrocannabinol. J. Pharm. Sci. 95, 1308-1317.

Herrmann, M. J., Römmler, J., Ehlis, A. C., Heidrich, A., and Fallgatter, A. J. (2004). Source localization (LORETA) of the error-relatednegativity (ERN/Ne) and positivity (Pe). Brain Res. Cogn. Brain Res. 20 , 294-299.

Hester, R., Nestor, L., and Garavan, H. (2009). Impaired error awareness and anterior cingulate cortex hypoactivity in chronic cannabis users. Neuropsychopharmacology 34 , 2450-2458.

Holroyd, C. B., and Coles, M. G. (2002). The neural basis of human error processing: reinforcement learning, dopamine, and the errorrelated negativity. Psychol. Rev. 109, 679-709.

Holroyd, C. B., Nieuwenhuis, S., Yeung, N., and Cohen, J. D. (2003). Errors in reward prediction are reflected in the event-related brain potential. Neuroreport 14, 2481-2484.

Holroyd, C. B., and Yeung, N. (2003). Alcohol and error processing. Trends Neurosci. 26, 402-404.
Horowitz-Kraus, T., and Breznitz, Z. (2009). Can the error detection mechanism benefit from training the working memory? A comparison between dyslexics and controls-an ERP study. PLoS ONE 4, e7141. doi: 10.1371/journal.pone.0007141

Hunault, C. C., Mensinga, T. T., Böcker, K. B., Schipper, C. M., Kruidenier, M., Leenders, M. E., de Vries, I., and Meulenbelt, J. (2009). Cognitive and psychomotor effects in males after smoking a combination of tobacco and cannabis containing up to 69 mg delta-9-tetrahydrocannabinol (THC). Psychopharmacology (Berl.) 204, 85-94.

Jentsch, J. D., Verrico, C. D., Le, D., and Roth, R. H. (1998). Repeated exposure to delta 9tetrahydrocannabinol reduces prefrontal cortical dopamine metabolism in the rat. Neurosci. Lett. 246 169-172.

Jocham, G., and Ullsperger, M. (2009). Neuropharmacology of performance monitoring. Neurosci. Biobehav. Rev. 33, 48-60.

Johannes, S., Wieringa, B. M., Nager, W. Dengler, R., and Münte, T. F. (2001). Oxazepam alters action monitoring. Psychopharmacology (Berl.) 155 100-106.

Kenemans, J. L., and Kähkönen, S. (2011). How human electrophysiology informs psychopharmacology: from bottom-up driven processing to top-down control. Neuropsychopharmacology 36 , 26-51.

Lane, S. D., Cherek, D. R., Tcheremissine, O. V., Lieving, L. M., and Pietras, C. J. (2005). Acute marijuana effects on human risk taking. Neuropsychopharmacology 30, 800-809.

Lansbergen, M. M., Dumont, G. J. H., van Gerven, J. M., Buitelaar, J. K., and Verkes, R. J. (2011). Acute effects of MDMA (3,4-methylenedioxymethamphetamine) on EEG oscillations: alone and in combination with ethanol or THC (delta9-tetrahydrocannabinol). Psychopharmacology (Berl.) 213, 745-756.

Larson, M. J., and Clayson, P. E. (2011). The relationship between cognitive performance and electrophysiological indices of performance monitoring. Cogn. Affect. Behav. Neurosci. 11 159-171.

Lorist, M. M., Boksem, M. A., and Ridderinkhof, K. R. (2005). Impaired cognitive control and reduced cingulate activity during mental fatigue. Brain Res. Cogn. Brain Res. 24 199-205.

Lupica, C. R., Riegel, A. C., and Hoffman, A. F. (2004). Marijuana and cannabinoid regulation of brain reward circuits. Br. J. Pharmacol. $143,227-234$

Luu, P., Flaisch, T., and Tucker, D. M. (2000). Medial frontal cortex in action monitoring. J. Neurosci. 20, 464-469.

Machado Rocha, F. C., Stéfano, S. C., De Cássia Haiek, R., Rosa Oliveira, L. M., and Da Silveira, D. X. (2008). Therapeutic use of Cannabis sativa on chemotherapy-induced nausea and vomiting among cancer patients: systematic review and meta-analysis. Eur. J. Cancer Care (Engl.) 170.17, 431-443.

Martín-Santos, R., Fagundo, A. B., Crippa, J. A., Atakan, Z., Bhattacharyya, S., Allen, P., Fusar-Poli, P., Borgwardt, S., Seal, M., Busatto, G. F., and McGuire, P. (2010). Neuroimaging in cannabis use: a systematic review of the literature. Psychol. Med. 40, 383-398.

McDonald, J., Schleifer, L., Richards, J. B., and de Wit, H. (2003). Effects of THC on behavioral measures of impulsivity in humans. Neuropsychopharmacology 28, 1356-1365.

Muntoni, A. L., Pillolla, G., Melis, M., Perra, S., Gessa, G. L., and Pistis, M. (2006). Cannabinoids modulate spontaneous neuronal activity and evoked inhibition of locus coeruleus noradrenergic neurons. Eur. J. Neurosci. 23, 2385-2394.

Nijboer, F., Birbaumer, N., and Kübler, A. (2010). The influence of psychological state and motivation on brain-computer interface performance in patients with amyotrophic lateral sclerosis - a longitudinal study. Front. Neurosci. 4:5 doi: 10.3389/fnins.2010.00055

Pailing, P. E., and Segalowitz, S. J. (2004). The effects of uncertainty in error monitoring on associated ERPs. Brain Cogn. 56, 215-233.

Pattij, T., Wiskerke, J., and Schoffelmeer, A. N. (2008). Cannabinoid modulation of executive functions. Eur. $J$ Pharmacol. 585, 458-463.

Penning, R., Veldstra, J. L., Daamen, A. P., Olivier, B., and Verster, J. C. (2010). Drugs of abuse, driving and traffic safety. Curr. Drug Abuse Rev. 3, 23-32.

Pistis, M., Ferraro, L., Pira, L., Flore, G., Tanganelli, S., Gessa, G. L., and Devoto, P. (2002). Delta(9)-tetrahydrocannabinol decreases extracellular GABA and increases extracellular glutamate and dopamine levels in the rat prefrontal cortex: an in vivo microdialysis study. Brain Res. 948 , 155-158.

Rabbitt, P. M. A. (1966). Errors and error correction in choice-response tasks. J. Exp. Psychol. 71, 264-272. 
Ramaekers, J. G.,Kauert, G., vanRuitenbeek, P., Theunissen, E. L., Schneider, E., and Moeller, M. R. (2006). Highpotency marijuana impairs executive function and inhibitory motor control. Neuropsychopharmacology 31, 2296-2303.

Ramaekers, J. G., Kauert, G., Theunissen, E. L., Toennes, S. W., and Moeller, M. R. (2009). Neurocognitive performance during acute $\mathrm{THC}$ intoxication in heavy and occasional cannabis users. J Psychopharmacol. 23, 266-277.

Ranganathan, M., and D'Souza, D. C. (2006). The acute effects of cannabinoids on memory in humans: a review. Psychopharmacology (Berl.) 188, 425-444.

Riba, J., Rodríguez-Fornells, A., Münte, T. F., and Barbanoj, M. J. (2005). A neurophysiological study of the detrimental effects of alprazolam on human action monitoring. Brain Res. Cogn. Brain Res. 25, 554-565.

Ridderinkhof, K. R., de Vlugt, Y., Bramlage, A., Spaan, M., Elton, M., Snel, J., and Band, G. P. (2002). Alcohol consumption impairs detection of performance errors in mediofrontal cortex. Science 298, 2209-2211.

Russo, E., and Guy, G. W. (2005). A tale of two cannabinoids: the therapeutic rationale for combining tetrahydrocannabinol and cannabidiol. Med. Hypotheses 66, 234-246.

Scheffers, M. K., Humphrey, D. G., Stanny, R. R, Kramer, A. F., and
Coles, M. G. H. (1999). Errorrelated processing during a period of extended wakefulness. Psychophysiology 36, 149-157.

Schellekens, A. F., De Bruijn, E. R. A., van Lankveld, C. A., Hulstijn, W., Buitelaar, J. K., de Jong, C. A., and Verkes, R. J. (2010). Alcohol dependence and anxiety increase error-related brain activity. Addiction 105, 1928-1934.

Schrijvers, D., De Bruijn, E. R. A., Maas, Y. J., Vancoillie, P., Hulstijn, W., and Sabbe, B. G. (2009). Action monitoring and depressive symptom reduction in major depressive disorder. Int. J. Psychophysiol. 71, 218-224.

Segalowitz, S. J., Santesso, D. L., Murphy, T. I., Homan, D., Chantziantoniou, D. K., and Khan, S. (2010). Retest reliability of medial frontal negativities during performance monitoring. Psychophysiology 47, 260-270.

Solowij, N., and Battisti, R. (2008). The chronic effects of cannabis on memory in humans: a review. Curr. Drug Abuse Rev. 1, 81-98.

Stemmer, B., Segalowitz, S. J., Witzke, W., and Schönle, P. W. (2004). Error detection in patients with lesions to the medial prefrontal cortex: an ERP study. Neuropsychologia 42, 118-130.

Sulzer, D., Sonders, M. S., Poulsen, N. W., and Galli, A. (2005). Mechanisms of neurotransmitter release by amphetamines: a review. Prog. Neurobiol. 75, 406-433.

Svrakic, D. M., Whitehead, C., Przybeck, T. R., and Cloninger, C. R. (1993). Differential diagnosis of personality disorders by the sevenfactor model of temperament and character. Arch. Gen. Psychiatry 50, 991-999.

Ullsperger, M., and von Cramon, D. Y. (2006). The role of intact frontostriatal circuits in error processing. J. Cogn. Neurosci. 18, 651-664.

Ullsperger, M., von Cramon, D. Y., and Müller, N. G. (2002). Interactions of focal cortical lesions with error processing: evidence from eventrelated brain potentials. Neuropsychology 16, 548-561.

Verrico, C. D., Jentsch, J. D., Roth, R. H., and Taylor, J. R. (2004). Repeated, intermittent delta(9)-tetrahydrocannabinol administration to rats impairs acquisition and performance of a test of visuospatial divided attention. Neuropsychopharmacology 29, 522-529.

Vicente, J., Olszewski, D., and Matias, J. (2008). "Prevalence, patterns and trends of cannabis use among adults in Europe," in A Cannabis Reader: Global Issues and Local Experiences. eds S. Rödner Sznitman, B. Olsson, and R. Room (Lisbon: European Monitoring Center on Drug and Drug Addiction), $1-26$.
Yeung, N., Botvinick, M. M., and Cohen, J. D. (2004). The neural basis of error detection: conflict monitoring and the error-related negativity. Psychol. Rev. 111, 931-959.

Zirnheld, P. J., Carroll, C. A., Kieffaber, P. D., O'Donnell, B. F., Shekhar, A., and Hetrick, W. P. (2004). Haloperidol impairs learning and error-related negativity in humans. J. Cogn. Neurosci. 16, 1098-1112.

Conflict of Interest Statement: The authors declare that the research was conducted in the absence of any commercial or financial relationships that could be construed as a potential conflict of interest.

Received: 31 May 2011; accepted: 24 August 2011; published online: 21 September 2011.

Citation: Spronk D, Dumont GJH, Verkes RJ and de Bruijn ERA (2011) Acute effects of delta-9-tetrahydrocannabinol on performance monitoring in healthy volunteers. Front. Behav. Neurosci. 5:59. doi: 10.3389/fnbeh.2011.00059

Copyright (c) 2011 Spronk, Dumont, Verkes and de Bruijn. This is an openaccess article subject to a non-exclusive license between the authors and Frontiers Media SA, which permits use, distribution and reproduction in other forums, provided the original authors and source are credited and other Frontiers conditions are complied with. 